\title{
CDISC Observation Class Terminology
}

National Cancer Institute

\section{Source}

National Cancer Institute. CDISC Observation Class Terminology. NCI Thesaurus. Code C82590.

The terminology subset that includes terms relevant to the Clinical Data Interchange Standards Consortium (CDISC) observation classes. 